\title{
Os indicadores bibliométricos: virtudes e limites no contexto da avaliação em Ciência e Tecnologia
}

\author{
Raimundo Nonato Macedo dos Santos \\ Doutor; Universidade Federal de Pernambuco, Recife, PE, Brasil; \\ rnmacedo@uol.com.br
}

\begin{abstract}
Resumo: O texto discute as bases necessárias para a formulação de Indicadores em Ciência e Tecnologia, suas virtudes e limites. Visto que quantificar é inerente ao ser humano, e que a aplicação dessa prática consistiu em um fator decisivo na construção do conhecimento e do expressivo sucesso das ciências exatas e das engenharias, já no século XIX, é inimaginável aceitar que não exista consenso sobre como medir e avaliar a produção intelectual e acadêmica. Nisso reside a motivação deste trabalho; na necessidade de uma reflexão sobre as propriedades gerais, natureza, gênese e efeitos da prática de quantificar em face da inexistência de consenso. Como opção, propõe-se a conceituar Indicadores em Ciência e Tecnologia de forma mais ampla, "como modelo". Assim, propõe-se um acordo, na concepção conceitual de "modelo", para medir e avaliar a produção intelectual e acadêmica, que subsidie, adequadamente e com equidade, tomadas de decisão, particularmente, referente à meritocracia e aos investimentos em pesquisa e inovação nos campos das ciências duras, humanas e aplicadas.
\end{abstract}

Palavras-chave: Indicadores em ciência e tecnologia. Avaliação da produção científica. Bibliometria.

Meu objetivo não é discutir o conteúdo da ciência nem tentar uma análise humanística de suas relações.

Derek J. de Solla Price, 1962

\section{Introdução}

Motivado pelas ideias de Derek de Solla Price que, expostas no dossiê "Aux sources de la scientométrie", de autoria de Xavier Polanco ${ }^{1}$, apresentam o modo segundo o qual o cientista formulou um modelo bibliométrico da ciência, publiquei, no ano de 2003, o artigo "Produção científica: por que medir? O que medir?". Nesse trabalho tratei, sobre todos os aspectos, o tema "indicadores quantitativos": a sua importância e caráter de interesse para especialistas e autoridades governamentais; o surgimento dos modelos bibliométricos e cientométricos, novos conceitos, aplicações e limitações; os sistemas de informação científica e técnica para a produção de indicadores; 
os aspectos metodológicos e ferramentas afins usadas no processo de produção de indicadores; a pesquisa, como uma vasta empresa de escritura que prolifera documentos de todos os tipos e reflexões sobre a complexidade dos sistemas; a carência de grupos de pesquisa aptos a compreender os fenômenos de criação do conhecimento e a transformá-los em resultados econômicos, a fim de identificar e estruturar, entender e interpretar os dados a serem apurados (SANTOS, 2003).

Relendo o artigo a partir de um enfoque distinto daquele do objeto a que ele se aplica, isto é, da produção científica, decidi desenvolver uma análise sobre as bases que antecedem a formulação dos indicadores em Ciência e Tecnologia (C\&T), suas virtudes e seus limites, na forma de um ensaio teórico.

Nessa perspectiva, torna-se inimaginável conceber a inexistência de um consenso a respeito do como medir e avaliar a produção intelectual e acadêmica, seus impactos e influências, sabendo-se que, além de ser um ato inerente ao ser humano, a quantificação teve participação decisiva na construção do conhecimento e do sucesso expressivo das ciências exatas e engenharias, já durante o século XIX (TRZESNIAK, 2014; OCDE $1993^{2}$ apud SPINAK, 1998). Ademais, segundo Moravcsik (1986³ apud SPINAK, 1998, p. 1), apesar de todo o esforço despendido para se estabelecer um conjunto de condições e classificações gerais, apropriado no que se refere à formulação dos indicadores em C\&T, esta é uma questão que está longe de ser resolvida.

Deste modo, a motivação para escrever este texto sobre indicadores nasceu da necessidade de desenvolver uma reflexão crítica sobre suas propriedades gerais natureza, gênese e efeitos - como recursos para melhor gerir, usar, analisar e interpretar resultados.

Os registros do Manual de Frascati, fornecem exemplos patentes para ilustrar esse estado de precariedade na implantação de bons indicadores em C\&T, aptos a proporcionar boas tomadas de decisões. Além disso, corroboram para justificar essa posição, posto que dão a dimensão da dificuldade que existe para se elaborar um sistema de indicadores em Ciência e Tecnologia:

Manual de Frascati, edição 1993:

Por definição, os indicadores representam um aspecto particular de um problema complexo, de múltiplas facetas. Faz-se necessário dispor de um modelo explícito que descreva de maneira mais precisa possível o siste- 


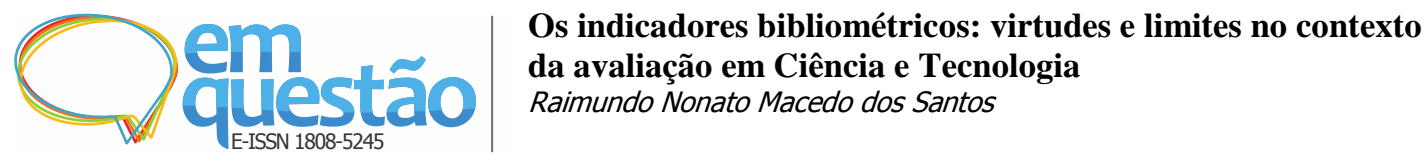

ma científico e a forma como ele se relaciona com a sociedade e com a economia. Na prática, e no estado natural das coisas, não existe um modelo explícito único capaz de estabelecer relações de causa entre a ciência, a tecnologia, a economia e a sociedade (OCDEM 1993, p. 3, grifo nosso, tradução nossa).

Manual de Frascati, edição 2002:

O presente Manual tem como objectivo medir os inputs da ID. A ID compreende tanto a ID contínua (formal) das unidades de ID como a ID ocasional (informal) de outras unidades. No entanto, o interesse em ID depende mais dos novos conhecimentos e inovações assim como dos efeitos económicos e sociais que deles derivam, do que da actividade em si. Infelizmente, enquanto que os indicadores dos resultados da ID são obviamente necessários para completar as estatísticas sobre os inputs, tais indicadores são muito difíceis de definir e de produzir. (OCDE, 2007, p. 25, grifo nosso). ${ }^{4}$

Entre outros exemplos nesse sentido, tem-se o registro do texto de Dainesi e Pietrobon (2007), um editorial, publicado na Revista Brasileira de Psiquiatria, que se manifestam de forma idêntica:

\footnotetext{
Decisões de mérito em ciência, seja relativa a um pesquisador ou grupo de pesquisadores ainda não são consensuais, e indicadores científicos de qualidade são adotados de forma universal. Do nosso ponto de vista, a questão que precisa ser colocada é sobre o processo como o indicador é formulado. Ciência é um processo complexo e essa complexidade não pode ser representada utilizando-se indicadores singelos (DAINESI; PIETROBOM, 2007, p. 101, tradução nossa, grifo nosso). ${ }^{5}$
}

Declarações como as apresentadas acima são encontradas com frequência na literatura, demonstrando que o referido assunto vem há muito sendo discutido e que desperta interesse de diversos pesquisadores, tendo como objeto ou contexto quase sempre seu próprio campo de atuação, e a preocupação com o uso de indicadores para decisões de fomento e estabelecimento de políticas em C\&T (MUELLER, 2008).

\section{Indicadores, quais são suas virtudes e limites? Conte sua história!}

Conceitos e definições de indicadores são raros e geralmente não dizem muito sobre do que se tratam. Assim, Stead (1976 ${ }^{6}$ apud HOLBROOK, 1992, p. 264) define indicadores em Ciência e Tecnologia como “[...] uma medida de um item usado para 
prover informação de outro item, imensurável [...]”, concluindo, em acordo de que C\&T são itens imensuráveis, que podem ser medidos através de indicadores, dos quais espera-se obter informações relevantes, não dedutíveis de forma trivial e inteligível por leigos - como, por exemplo, tomadores de decisão.

Refletindo também sobre questões dessa ordem, Mueller (2008, p. 4) indagase: "Mas o que são indicadores e métricas no contexto da avaliação da ciência e da tecnologia?", recorrendo, em seguida, a Geisler (2000), que oferece definição para ambos os termos:

[...] define indicadores da ciência como um termo genérico que se aplica a um amplo espectro de medidas quantitativas utilizadas para medir atividades, insumos, e resultados da pesquisa, desenvolvimento e inovação; e define o termo métricas como um sistema de medidas que inclui o item objeto da medida, a unidade de medida e o valor da unidade. Geisler classifica as métricas como objetivas ou subjetivas. A avaliação pelos pares é citada pelo autor como uma métrica subjetiva e a contagem de patentes como uma métrica objetiva. Ainda de acordo com a definição de Geisler, as métricas podem tomar vários formatos, por exemplo, uma medida única, uma razão (entre duas medidas), um índice, ou ainda uma medida integrada que combine várias métricas, até mesmo com atributos diferentes, objetivos e subjetivos (MUELLER, 2008, p 27).

Mas o que há de essencial nessas sentenças? Quais são as virtudes e os limites dos indicadores em C\&T? Para mim, são questões não abarcadas pelas definições acima, e que são objeto, portanto, das motivações para a realização deste ensaio. Como opção, entendendo que, para refletir sobre questões dessa ordem, se faz necessário conceituar o indicador em C\&T numa acepção mais ampla, isto é, como um modelo (TRZESNIAK, 1998). Para esse autor, um modelo consiste de:

[...] um procedimento de qualquer natureza (prático, matemático, gráfico, verbal...) capaz de, em todos os aspectos relevantes, reproduzir uma relação de antecedentes (causas) e consequentes (efeitos) de forma idêntica como essa relação ocorre no universo em que estamos inseridos. (TRZESNIAK, 1998, p. 159, grifo nosso).

O que de essência encerra, para os meus propósitos, esse axioma? A sentença anterior encerra duas expressões: "relação" e "de forma idêntica". Pensar sobre a dimensão da complexidade que guarda o estabelecer "relações entre causa e efeito", bem como sobre as grandezas, padrões utilizados no procedimento que promove a correlação do modelo com o fenômeno, são reflexões necessárias que antecedem a 


\section{formulação do indicador. Essa é a condição sine qua non que melhor possibilita} a compreensão da seriedade e da dimensão da responsabilidade a cerca do estabelecimento de um consenso e da formulação de indicadores em C\&T de qualidade, aptos à produção de resultados adequados e seguros para avaliar e permitir a tomada de decisões com equidade a respeito de investimentos em pesquisa e inovações nos campos de conhecimento puros ou aplicados.

Desse ponto de vista, refletir sobre o desafio da implementação desse vínculo, através de padrões de referência - grandezas físicas, matemáticas -, do modelo e daquilo que ele se propõe a representar, requer acuidade sobre o espaço de jogo de interesses onde opera o estabelecimento de padrões. Área de exata contemplada, mesmo com todo o entusiasmo de William Thompson, o famoso Lord Kelvin, que, há mais de 150 anos dizia:

\footnotetext{
Afirmo muitas vezes que, se você medir aquilo de que está falando e o expressar em números, você conhece alguma coisa sobre o assunto; mas, quando você não o pode exprimir em números, seu conhecimento é pobre e insatisfatório; pode ser o início do conhecimento, mas dificilmente seu espírito terá progredido até o estágio da Ciência, qualquer que seja o assunto. (RESNICK; HALLIDAY, $1974^{7}$ apud TRZESNIAK, 1998, p. 159).
}

Registros históricos de toda ordem dão prova de que a instauração de grandezas físicas na área de ciências exatas - por exemplo, medida de temperatura - , por mais simples que nos pareça, por trás dessa grandeza existem sempre jogos de interesses quando há aplicação prática desta para um determinado fim, anacronismos e preconceitos, por vezes insuperáveis, que geram prejuízos de toda ordem, gerando, por exemplo, catástrofes ambientais como o absurdo efeito estufa.

Para ilustrar, exemplifico com um projeto com o qual estive envolvido no Instituto de Pesquisas Tecnológicas de São Paulo, no auge da crise do petróleo, final dos anos 70. O projeto tinha como objetivo desenvolver um programa para nacionalizar um conjunto de Normas Brasileiras.

No caso de fabricação de telhas, a Norma Brasileira em vigor na época, baseada na Norma Europeia - Association Française de Normalisation (AFNOR) -, especificava o índice de absorção de água pela telha como igual a zero, valor que correspondia a "Grandeza Padrão", portanto, uma abstração estabelecida para o fe- 
nômeno "absorção de água pela telha" para uma telha ser tida como impermeável. Para satisfazer essa especificação, são necessárias temperaturas elevadíssimas, que exigem o consumo excessivo de lenha nos fornos cerâmicos, destruindo assim as florestas que, na época, eram a base energética que o país dispunha para esse fim. O problema do desperdício era evidente, uma vez que um país como o nosso que, salvo raríssimas exceções, não atinge temperaturas abaixo de zero - situação na qual a água, ao passar do estado líquido para o sólido, se expandiria provocando a quebra da telha -, desqualifica todo o sentido de atender o padrão estabelecido pela Norma em vigor.

Assim, mesmo com tantas evidências favoráveis à mudança, somente uma situação de crise bastaria para vencer o jogo de interesses e fazer com que os protagonistas adotassem, de forma consensual, os novos padrões. Dentre estes, estavam inclusos toda a cadeia de produção de telha, o fornecedor da lenha, o fabricante de forno, o fornecedor da matéria prima, os pesquisadores, os fabricantes de instrumentos de controle de fornos cerâmicos, os consumidores etc. Tudo somente após pesquisas sobre as características gerais da nova telha, elaboração do projeto de norma, aprovação da norma e assim por diante.

Exemplos dessa ordem poderiam ser citados ad infinitum. Embora tudo seja muito lógico e racional ao tratar-se dos fenômenos físicos que, por serem prioritariamente determinísticos, com suas relação causa-efeito estabelecida de forma direta, ideais para a quantificação e modelagem matemática, pode-se assegurar que, no entanto, não são de fácil apropriação - admitindo aqui a diferença entre conhecer e agir. Isso justifica o fato de, na atualidade, considerarmos um tanto quanto exagerado o entusiasmo do Lord Kelvin (TRZESNIAK, 2008).

Se o esforço requerido para o estabelecimento de padrões, de Normas, de grandezas na área das exatas assume proporções dessa ordem, por inferência, justifica-se a cautela na manifestação da OCDE, ao considerar o fato de não existir consenso sobre como medir e avaliar a produção intelectual e acadêmica, nem na interpretação de seus impactos e influências (OCDE, 1993², apud SPINAK, 1998). Some-se a isso o fato de se tratarem de indicadores em C\&T, ou seja, de estudos para quantificar as atividades científicas compreendidos em uma Ciência das Ciências, da qual consiste a área de ciências sociais. Não é sem razão que, dessa forma, a área das 
ciências sociais, semelhantemente às ciências humanas, é considerada pela sua comunidade científica como um ambiente bem menos propício à produção de indicadores quantitativos.

\subsection{Indicadores em Ciência \& Tecnologia}

A ciência, como atividade, é uma complexa construção social de conhecimento objetivado. Reconhecidamente, trata-se de um processo de natureza não determinística e, como tal, requer modelos de vinculação da relação causa e efeito de natureza idêntica; modelos estocásticos, em que a vinculação entre causa e efeito torna-se indireta, isto é, a presença - ou variação - da primeira reflete-se não no efeito, mas na probabilidade de ele surgir ou se modificar (TRZESNIAK, 1998). No ambiente de construção de conhecimento as ações e o comportamento dos cientistas dependem do contexto; não são, portanto, nem neutros, nem extemporâneos: as ações prosperam na concentração do labor representado, fundamentalmente pela produção científica certificada que, em decorrência, favorece, com os seus resultados, o monopólio econômico (SANTOS, 2003). Elitiza-se e materializa-se no "efeito São Mateus", que diz que a quem tem, mais lhe será dado (KROPF; LIMA, 1999).

A ciência evolui por meio da pesquisa, uma vasta empresa de escritura que produz documentos de todos os tipos. No campo científico, três funções parecem ser básicas: disseminar conhecimentos; assegurar a preservação de padrões; e atribuir créditos e reconhecimento para aqueles cujos trabalhos têm contribuído para o desenvolvimento científico em diferentes campos (MACIAS-CHAPULLA, 1998).

Do ponto de vista da materialização da dinâmica desse processo, tem-se o seguinte postulado:

[...] uma obra científica é o produto objetivado da atividade intelectual criativa. No contexto científico, uma publicação é a representação da atividade de pesquisa. $\mathrm{O}$ maior esforço do autor é o de persuadir os pares de que suas descobertas, seus métodos e técnicas são particularmente pertinentes. A comunicação escrita fornecerá, portanto, os elementos técnicos, conceituais, sociais e econômicos que o autor busca afirmar ao longo de sua argumentação (ROSTAING, 1996, p. 21). 
Por essa razão, sem entrar no mérito do conteúdo ou de relações humanísticas, estudar, do ponto de vista quantitativo, os elementos técnicos, conceituais, sociais e econômicos que os autores buscam afirmar ao longo de suas argumentações, nos permite vincular "quantitativamente" essas grandezas - aspectos relevantes às suas estruturas, segundo as escolas de pensamento e suas evoluções, correspondendo assim a uma simplificação do evento real sob a forma de Indicadores em C\&T.

Considerando o que foi ressalvado anteriormente, tal assertiva sustenta a proposição de que elementos relevantes expostos nas publicações científicas e patentes, por exemplo, constituem fontes de informação e assumem papel destacado e inconteste de indicadores de produção de conhecimentos certificados no domínio das ciências e das técnicas (POLANCO, 1995 apud SANTOS, 2003). Ademais, as manifestações de Derek de Solla Price ao escrever o prefácio do seu livro "Little science, big science", traduzido para o português como o "Desenvolvimento da ciência", faziam sentido e continham bases objetivas e passíveis de realização:

\footnotetext{
Meu objetivo não é discutir o conteúdo da ciência nem tentar uma análise humanística de suas relações. Em vez disso, quero esclarecer essas abordagens mais comuns, considerando separadamente todas as análises científicas que se podem fazer da ciência. Por que não empregar os instrumentos da ciência à própria ciência? Por que não medir e generalizar, levantar hipóteses e tirar conclusões? (PRICE, 1976, p. 21).
}

Desse modo, essas bases foram um domínio de especialidade em nada recente, conjugadas a máquinas com recursos inteligentes - particularmente os computadores - para guardar, compilar, organizar e reorganizar informações, que favoreceram o desenvolvimento de pesquisas para formulação e uso de indicadores quantitativos de produção científica na contemporaneidade, outorgando importância crescente como instrumentos para análise da atividade científica e das suas relações com o desenvolvimento econômico e social.

\section{Estudos métricos da informação}

O uso de métodos estatísticos e matemáticos para mapear informações não é fato novo, como aponta Boustany (1997) ao se referir a um autor desconhecido, citado 
no "Manuel du Bibliophile ou, traité du choix des livres", de 1823, de autoria de Gabriel Peignol, que pesquisou a produção universal de livros no período compreendido entre a metade do século XV e início do século XIX.

Lotka, Bradford, Zipf e Price são os autores que, no século XX, contribuíram de forma mais significativa na consolidação do campo dos estudos quantitativos sobre a informação. A lei de Lotka (1926) sobre a produtividade de autores de artigos científicos, a lei de Bradford (1934) sobre a dispersão de autores em publicações periódicas, e a lei de Zipf (1935), sobre a frequência/ocorrência de palavras em textos longos, são ainda hoje parâmetros utilizados nos estudos métricos da informação.

Price, por sua vez, em 1969, se valeu das propostas de Lotka, Bradford e Zipf para formular a Cientometria, caracterizada como o estudo quantitativo da atividade científica cujos objetos empíricos são os produtos e os produtores de ciência. Uma caracterização semelhante teve como ponto de partida a percepção de que as leis econométricas relativas ao cálculo de mão de obra poderiam explicar o comportamento da literatura científica. Com base nesse raciocínio, Price afirmou que os dados quantitativos sobre revistas e artigos científicos obedecem a regras estáveis que operam como indicadores do estado da ciência (PRICE, 1969).

Segundo Meadows $\left(1990^{8}\right.$ apud POLANCO, 1995):

\begin{abstract}
Price foi a figura chave destes novos estudos quantitativos, cujos escritos, especialmente Little Science, Big Science tiveram o maior impacto nas concepções sobre o crescimento e a evolução das revistas científicas. Em parte, ele reuniu ideias já em discussão. Por exemplo, o rápido crescimento na quantidade de literatura científica havia sido debatido por bibliotecários, e outros pesquisadores, desde a Primeira Guerra Mundial. Da mesma forma, mas separadamente, havia os trabalhos sobre distribuições estatísticas relevantes, como o de Lotka sobre a produtividade científica e a de Zipf sobre as distribuições de palavras. Price ampliou esses trabalhos pioneiros de modo a dar uma visão integrada, um quadro quantitativo da literatura científica [...] Uma área importante do trabalho de Price são as aplicações da análise de citações. Neste caso, ele se baseou nas atividades de Garfield de desenvolvimento do conceito de índice de citação (MEADOWS, 1990 apud POLANCO, 1995, doc. sem paginação, tradução nossa).
\end{abstract}

Deve-se a Price, portanto, a elaboração de um novo modelo que integrou Bibliometria e estudos da atividade científica, ou seja, a formulação teórica da Ciência da Ciência, campo que propiciou o desenvolvimento de importantes estudos interdisciplinares entre Cientometria e Ciência da Informação. Da mesma forma, Small e 
Garfield (1986) afirmaram que Price antecipou, nos anos 60, a ideia de evidenciar o grau de cobertura e a importância relativa das revistas científicas por meio de mapas da ciência. Os referidos mapas têm por base, segundo Price $\left(1965^{9}\right.$ apud POLANCO, 1995, doc. sem paginação) em seu texto "Is Technology historically independent of Science? A study in Statistical Historiography", as "relações estruturais de uma rede de referências e citações", projeto que foi desenvolvido no Institute for Scientific Information (ISI), na década de 70, por meio de métodos de cocitação.

Como se pode verificar com base no postulado de trabalho subjacente às análises quantitativas dos produtos da atividade científica e técnica, os modelos bibliométricos e cientométricos utilizam artigos científicos para examinar a atividade científica e realizar inferências sobre o estado da ciência. Procedimentos que somente ratificam as diversas manifestações apresentadas neste ensaio, dando conta de que os modelos tradicionais têm como foco a quantificação de artigos, de autores mais produtivos, de periódicos mais citados, etc.; indicadores que favorecem a meritocracia, referendam privilégios, validando o "efeito Mateus" sem, no entanto, penetraram nas questões cognitivas, ou seja, no conhecimento contido nesses objetos, conforme nos adverte Polanco:

[...] o modelo bibliométrico de ciência de Price não captura o conteúdo cognitivo dos artigos científicos, ou seja, o conhecimento que eles transmitem. As informações presentes no título do artigo, em seu resumo, ou no próprio texto são, na realidade, ignoradas na abordagem cientométrica introduzida por Price [...] Portanto, tal dispositivo realiza uma cientometria “externalista". [...] (POLANCO, 1995, doc. sem paginação, tradução nossa).

Os estudos métricos das atividades da ciência, formulados por Price (1962), cujos objetos empíricos são os produtos e produtores da ciência, constituem-se, pois, de um "reducionismo bibliométrico"; uma visão cumulativa da ciência - leia-se "efeito Mateus" -; uma proposta de uma Ciência da Ciência. Ainda sobre esse propósito, Price (1962) sugere que "o grau de utilização parece ser o melhor teste de qualidade" - leia-se indicadores de citação, obtidos pela contagem do número de citações recebidas por um periódico ou artigo de periódico -, "do que o simples critério da 'quantidade produzida' obtida pela contagem do número de publicações por tipo de documento (livros, artigos, publicações científicas, relatórios etc.), por insti- 
tuição, área de conhecimento, país” (PRICE, $1962^{10}$ apud POLANCO, 1995, doc. sem paginação).

De acordo com sua tese, Price advogava que "A quantidade de utilização fornece uma medida razoável do grau importância científica de um periódico ou da produção de um indivíduo.” (PRICE, $1963^{11}$ apud POLANCO, 1995, doc. sem paginação).

[...] concentrou seus estudos sobre a utilização, - valor de uso -, dos periódicos científicos, que pode ser formulada a partir da curva estatística obtida pela distribuição de frequência dos acessos aos títulos dos periódicos dispostos em ordenada em relação aos títulos dos periódicos acessados em abscissa. Essa curva apresenta-se em acordo à Lei de Bradford (POLANCO, 1995, doc. sem paginação).

May (1967 ${ }^{12}$ apud MACIAS-CHAPULA, 1998, p. 136) contesta essa visão ortodoxa de que as citações oferecem um quadro preciso das relações intelectuais entre as publicações, e conclui que: “[...] os autores selecionam citações para servir a seus objetivos científicos, políticos, e não para descrever sua linha intelectual”. As classificações e abordagens diversas sobre processos e comportamentos de citação constituem uma questão polêmica que tem sido objeto de estudos em muitas pesquisas nas últimas décadas, a maioria tendendo a contestar os padrões defendidos por Price.

Polanco (1995), no dossiê citado no início deste ensaio sobre os princípios que conduziram Price a formular um modelo bibliométrico para a Ciência, é também um crítico ao procedimento adotado, propondo uma nova orientação ou, preferencialmente, uma mudança de paradigma: o projeto de uma engenharia de conhecimento que abarque métodos infométricos, termo adotado em 1987 pela International Federation of Documentation - FID, para designar o conjunto das atividades métricas relativas à informação, cobrindo tanto a bibliometria quanto a cientometria. Nesse sentido, alarga o conceito de modelo:

[...] uma equação ou uma estrutura (quando não se trabalha com um modelo numérico) destinado a representar, de uma maneira simplificada porém consistente, um procedimento ou um sistema. A construção de um modelo estatístico está destinada a analisar, prever ou decidir a partir de uma base rigorosa e confiável POLANCO, 1995, doc. sem paginação, tradução nossa) $)^{13}$. 
Essa nova dimensão de modelo conseguirá abarcar o desenvolvimento de uma cientometria "internalista", se assim se pode falar, com base no que já vinha sendo feito na França, desde o início dos anos 80, por meio da análise de palavras associadas, tendo as palavras-chave como indicadores de conhecimento. Os indicadores de ligação, por sua vez, são construídos pela análise de coocorrência de autorias, citações e palavras, sendo aplicados na elaboração de mapas de estruturas de conhecimento e de redes de relacionamento entre pesquisadores, instituições e países. Além disso, sua elaboração requer o emprego de técnicas de análise estatística de agrupamentos (COURTIAL,1990; CALLON; COURTIAL; PENAN, 1993; NARIN; OLIVASTRO; STEVENS, 1994; OKUBO, 1997; MACIAS-CHAPULA， 1998; SPINAK, 1998).

Essas novas análises se sustentam com base no segundo postulado de trabalho, subjacente às análises quantitativas dos produtos da atividade científica e técnica, que pressupõe:

[...] a atividade de publicação científica é uma eterna confrontação entre as reflexões intrínsecas do autor e os conhecimentos que ele adquiriu pela leitura dos trabalhos originários dos outros autores. Desta forma, a publicação é o resultado de uma comunicação entre a razão individual e a coletiva. Assim, os pesquisadores, para consolidar suas argumentações, fazem referência aos trabalhos dos outros pesquisadores que, constituem, com esse arranjo, um certo consenso na comunidade científica. Deste fenômeno, pode-se dizer que: existe uma relação entre todos os trabalhos científicos publicados, não sendo possível, no entanto, precisar o tipo de relação: se direta ou indireta, reconhecida ou dissimulada, consciente ou inconsciente, acordada ou não (ROSTAING, 1996, p. 21).

O cálculo de coocorrências (de autores, de palavras, de instituições), fundado em métodos de análise multidimensional, é uma das áreas que vem crescendo de forma acentuada. A análise multidimensional aplicada às palavras-chave de registros bibliográficos configura-se como uma das mais recentes contribuições teóricas aos métodos quantitativos. Baseado no cálculo matricial e na álgebra linear, esse método supõe a classificação automática dos dados e sua representação por meio de cartografias temáticas. 


\section{Considerações finais}

Concebida como uma construção social do conhecimento objetivado, uma atividade dinâmica em permanente mutação e de execução complexa e intricada, a Ciência constitui-se, por excelência, como um processo de natureza não determinístico, e como tal, requer modelos de vinculação da relação causa e efeito de natureza idêntica, isto é, modelos estocásticos, em que a vinculação entre causa e efeito torna-se indireta, e a presença - ou variação - da primeira reflete-se não no efeito, mas na probabilidade de ele surgir ou se modificar (TRZESNIAK, 1998).

Assumindo a atividade científica, particularmente no campo das ciências humanas e sociais, como processo de natureza estocástica, advogamos que a prática de processos de tais dimensões com o propósito de obter resultados adequados e seguros para avaliar e permitir tomadas de decisão com equidade sobre investimentos em pesquisa e inovação nos campos de conhecimento puros e aplicados, constitui uma das metas mais importantes na busca pelo conhecimento quantificado.

Segundo essa perspectiva, embora não se concorde totalmente com o entusiasmo do Lord Kelvin a respeito da quantificação do conhecimento, não cabe renegálo sumariamente. É inquestionável que, ao se conseguir expressar aquilo de que se está falando em números, de forma quase recorrente, o conhecimento será mais rico e mais satisfatório. Para tanto, constitui-se o desafio de monta que requer: uma concepção prévia de modelos nos moldes acima propostos; uma coleta sistemática; o entendimento do contexto em que se está inserido; e, por fim, a correta interpretação dos indicadores relativos ao fenômeno ou processo objeto do estudo.

\section{Agradecimento}

O autor agradece ao Conselho Nacional de Desenvolvimento Científico e Tecnológico $(\mathrm{CNPq})$ pela bolsa de produtividade concedida a Raimundo Nonato Macedo dos Santos. 


\section{Referências}

BOUSTANY, J. La production des imprimés non-périodiques au Liban de 1733 à 1920: étude bibliométrique. 1997. Tese (Doutorado em Sciences de l'Information et de la Communication) - Université Michel de Montaigne Bordeaux III, Bordeaux, 1997.

CALLON, M.; COURTIAL, J-P.; PENAN, H. La scientométrie. Paris: Presses Universitaires de France, 1993. (Que sais-je? 2727).

COURTIAL, J. P. Introduction à la scientométrie: de la bibliométrie à la veille technologique. Paris: Anthropos, 1990.

DAINESI, S. M.; PIETROBON, R. Scientific indicators of productivity: time for action. Revista Brasileira de Psiquiatria, São Paulo, v. 29, n. 2, jun., 2007. Disponível em: 〈http://dx.doi.org/10.1590/S1516-44462007000200002〉. Acesso em: 7 set. 2015 .

GEISLER, E. The metrics of science and technology: evaluation and measurement of research, development, and innovation Westport, CT: Quorum Books, 2000.

HOLBROOK, J. A. D. Why measure science? Science and public policy, Oxford, v. 19 , n. 5, p. 262-266, out., 1992.

KROPF, S. P.; LIMA, N. T. Os valores e a prática institucional da ciência: as concepções de Robert Merton e Thomas Kuhn. História, Ciências, Saúde-

Manguinhos, Rio de Janeiro, v. 5, n. 3, fev. 1999. Disponível em:

<http://dx.doi.org/10.1590/S0104-59701999000100002 >. Acesso em: 7 set. 2015.

MACIAS-CHAPULA, C. A. O papel da informetria e da cienciometria e sua perspectiva nacional e internacional. Ciência da Informação, Brasília, v. 27, n, 2, p. 134-140, maio/ago., 1998.

MORAVCSIK, M. J. The classification of science and the science of classification. Scientometrics, Amsterdam, v. 10, n. 3-4, p. 179-97, 1986.

MUELLER, S. P. M. Métricas para a ciência e tecnologia e o financiamento da pesquisa: algumas reflexões. Encontros Bibli, Florianópolis, v. 13, n. esp., p. 24-35, 2008. Disponível em:

<https://periodicos.ufsc.br/index.php/eb/article/download/15182924.2008v13nesp1p24/1593>. Acesso em: 7 set. 2015.

NARIN, F.; OLIVASTRO, D.; STEVENS, K. A. Bibliometrics/theory, practice and problems. Evaluation Review, New York, v. 18, n. 1, p. 65-76, 1994.

OCDE. Manual de Frascati: Proposta de práticas exemplares para inquéritos sobre investigação e desenvolvimento experimental 2002. Coimbra: OCDE, 2007. 
OCDE. Manual de Frascati: propuesta de norma práctica para encuestas de investigación y desarrollo experimental. Paris: OCDE, 1993.

OKUBO, Y. Bibliometric indicators and analysis of research systems: methods and examples. Paris: OCDE, 1997. Disponível em: <http://dx.doi.org/10.1787/208277770603>. Acesso em: 7 set. 2015.

POLANCO, X. Aux sources de la scientométrie. Solaris, Rennes, n. 2, 1995. Disponível em: 〈http://gabriel.gallezot.free.fr/Solaris/d02/2polanco1.html〉. Acesso em: 7 set. 2015 .

PRICE, D. J. de S. O desenvolvimento da ciência: análise histórica, filosófica, sociológica e econômica. Rio de Janeiro: Livros Técnicos e Científicos Editora S. A., 1976.

PRICE, D. J. de S. The structures of publication in science and technology. In: GRUBER, H.; MARQUIS, D.G. (Ed.). Factors in the transfer of technology. Cambridge: MIT Press, 1969. p. 91-104.

ROSTAING, H. La bibliométrie et ses techniques. Tolouse: Sciences de la Société, 1996.

SANTOS, R. N. M. Produção científica: por que medir? O que medir? Revista Digital de Biblioteconomia e Ciência da Informação, Campinas, v. 1, n. 1, p. 22-38, jul./dez., 2003.

SMALL, H.; GARFIELD, E. The geography of science: disciplinary and national mappings. Journal of Information Science, v. 11, n. 4, p. 147-159, 1986.

SPINAK, E. Indicadores cienciométricos. Ciência da Informação, Brasília, DF, v. 27, n. 2, p. 141-148, 1998.

TRZESNIAK, P. Indicadores quantitativos: como obter; avaliar; criticar e aperfeiçoar. Navus - Revista de Gestão e Tecnologia, Florianópolis, v. 4, n. 2, p. 5-18, jul./dez., 2014.

TRZESNIAK, P. Indicadores quantitativos: reflexões que antecedem seu estabelecimento. Ciência da Informação, Brasília, v. 27, n. 2, p. 159-164, maio/ago., 1998.

Bibliometric indicators: virtues and limits in the context of Science \& Technology assessment. 


\begin{abstract}
This essay presents a reflection on the necessary bases preceding the formulation of Science and Technology (S\&T) Indicators by addressing their virtues and limits. Quantifying is inherent in human nature and the application of this practice in the building knowledge processes was a decisive factor for the significant success of exact sciences and engineering in the $19^{\text {th }}$ century; therefore, it is unthinkable that in contemporary times there is no consensus on how to measure and assess intellectual and academic production. Thus, the motivation for writing this paper lies with the need to reflect critically on S\&T Indicators' general properties, nature, genesis and effects, given the inexistence of such a consensus. In order to address this issue, it is proposed to conceptualize S\&T Indicators in a broader sense, namely as a model, considering that, historically, this one of the most important goals in the quest for knowledge, for both, producing adequate and safe results, and enabling equity evaluation and decision-making on investments in research and innovation in applied and pure sciences.
\end{abstract}

Keywords: Science and technology Indicators. Evaluation of scientific production. Bibliometrics.

Recebido em 11/09/2015

Aceito em 04/12/2015

\footnotetext{
${ }^{1}$ Engenheiro pesquisador junto ao Grupo de Infometria do l'Institut de l'Information Scientifique et Technique do Centre National de la Recherche Scientifique - INIST-CNRS, Nancy, França, publicado no Dossier Solaris, n. 2: Bibliométrie, Scientometrie, Infométrie. (POLANCO, 1995).

${ }^{2}$ OCDE. Manual de Frascati: propuesta de norma práctica para encuestas de investigación y desarrollo experimental. Paris: OCDE, 1993.

${ }^{3}$ MORAVCSIK, M. J. The classification of science and the science of classification. Scientometrics, v. 10, n. 3-4, p. 179-97, 1986.

${ }^{4}$ No original: Por definición los indicadores ilustran un aspecto particular de una cuestión compleja y de facetas múltiples. Es necesario disponer de un modelo explícito que describa a la vez el sistema científico en sí mismo y la forma en que se relaciona con el resto de la sociedad y con la economía. En la práctica y en el estado actual de cosas, no existe un modelo explícito único capaz de establecer relaciones causales entre la ciencia, la tecnología, la economía y la sociedad (OCDE, 1993, p. 3, grifo nosso).
} 
${ }^{5}$ No original: Decisions about science based on merit of individual or groups of researchers need to be made, and scientific indices of quality are the natural means by which this is accomplished. The problem, we believe, is in how this is accomplished. Science is a very complex construct and its complexity will not be mapped using single indicators (DAINESI; PIETROBOM, 2007, p. 101, grifo nosso).

${ }^{6}$ STEAD, H. Costs of technological innovation. Research Policy, v. 5, n. 1, p. 209, 1976.

${ }^{7}$ RESNICK, R.; HALLIDAY, D. Física. 2. ed. Rio de Janeiro: Livros Técnicos e Científicos, 1974. V. 1.

${ }^{8}$ MEADOWS, A. J. Theory in information science. Journal of Information Science, New York, v. 16, n. 1, p. 59-63, 1990.

${ }^{9}$ PRICE, D. J. de S. Is technology historically independent of science? A statistical historiography. Technology and Culture, Baltimore, v. 6, n. 4, p. 553-568, 1965.

${ }^{10}$ PRICE, D. J. de S. Science since Babylon. New Haven: Yale University, 1962.

${ }^{11}$ PRICE, D. J. de S. Little science, big science. New York: Columbia University, 1963.

${ }^{12}$ MAY, K. O. Abuses of citation indexing. Science, n. 156, p. 890-2, 1967.

13 No original: [...] une équation ou une structure (quand on ne travaille pas avec un modèle numérique) destinée à représenter, d'une manière simplifiée mais consistante, un processus ou un système. La construction d'un modèle statistique est destinée à analyser, prévoir ou décider à partir d'une base rigoureuse et fiable (POLANCO, 1995, doc. sem paginação). 